\title{
Electron interactions, classical integrability, and level statistics in quantum dots
}

\author{
Lilia Meza-Montes and Sergio E. Ulloa \\ Department of Physics and Astronomy, Condensed Matter and Surface Science Program, Ohio \\ University, Athens, $\mathrm{OH}$ 45701-2979, USA \\ Daniela Pfannkuche \\ Max-Planck Institut für Festkörperforschung, Heisenbergstr. 1, D-70569 Stuttgart, Germany
}

(August 28, 1997)

\begin{abstract}
The role of electronic interactions in the level structure of semiconductor quantum dots is analyzed in terms of the correspondence to the integrability of a classical system that models these structures. We find that an otherwise simple system is made strongly non-integrable in the classical regime by the introduction of particle interactions. In particular we present a twoparticle classical system contained in a $d$-dimensional billiard with hard walls. Similarly, a corresponding two-dimensional quantum dot problem with three particles is shown to have interesting spectral properties as function of the interaction strength and applied magnetic fields.
\end{abstract}

Keywords: Quantum dots, chaos, level statistics

Sergio E. Ulloa

E-mail: sulloa1@ohiou.edu

Fax: (614) 593-0433 


\section{INTRODUCTION}

The role of electronic interactions on the observed properties of semiconductor quantum dots has increasingly been found to be of vital importance, as many of the papers in these proceedings and elsewhere illustrate. More and more, transport and capacitance experiments, as well as detailed studies of far-infrared response, require the consideration of interactions in order to understand the observed experimental features. Since a number of experiments explore specifically the electronic level spectrum, it is of interest to contrast these studies with a detailed theoretical analysis of the role of interactions. We present here an analysis of the energy level statistics of a quantum dot system as function of variable interaction strength, and then as function of an applied magnetic field.

As the level spectrum is considered in detail, the correspondence with the dynamical integrability of a classical system is also of interest. It is anticipated that as the Coulomb interaction is introduced, the dynamics would in general become chaotic and this would in turn be reflected in various statistical properties of the level spectrum. It has been known for some time now that as a classical system becomes non-integrable [1], the corresponding quantum system exhibits a level spacing distribution given by the 'Wigner surmise' derived in the context of random matrix theory (RMT) [2]. In fact, this behavior has been verified in a number of theoretical and experimental systems, although typically the classical nonintegrability is due to disorder or boundary (geometrical) effects. Examples discussed in the literature include small disordered metallic particles [3], particles moving in a variety of 'stadia' [4,5], and in two-dimensional antidot arrays [6 8]. RMT also has been used to make definite predictions on the statistical distribution of Coulomb blockade peak amplitudes [9]. This behavior has in fact been shown recently to be a good description of quantum dot systems in beautiful experimental realizations [10]. One should also mention that recent work on interacting systems, some without an obvious classical counterpart, has also shown that these exhibit the level repulsion characteristic of quantum versions of non-integrable classical systems [11]. Moreover, recent analysis of the level spectrum of excitons in quantum 
wells (via photoluminescence excitation spectroscopy) has shown evidence of level repulsion [12.

A level structure described by RMT has been sought recently in the charging (or addition) spectrum of quantum dots [13]. Detailed analysis of experimental charging energies, after proper subtraction, would be expected to yield the single-particle-like excitation spectrum described by RMT. Unfortunately, it appears that the extraction of this excitation spectrum is obscured by the systematic shift in the charging energies, and the resulting level spacing is described by a normal distribution, rather than by the RMT functions. This would then suggest that a more direct measurement of the excitation spectrum (via perhaps nonlinear tunneling currents) would be desirable (although clearly difficult experimentally beyond the first few excitations [14]). One should also mention here that it is believed that the nonlinear transport experiments explore mainly the excitations of the center of mass of the system (in the typically parabolic quantum dots) [15], due to the strong electronic correlations suppressing most of the tunneling 'channels'. This prevalence of the center of mass excitations is however expected theoretically to diminish as the energy of the excitation increases [15]. This regime would be reached only as the bias voltage is raised in transport experiments, and makes it then difficult to achieve in practice. We hope, however, that the results presented here would motivate more experimental work in this direction.

\section{A CLASSiCAL DOT}

We have studied the effects of particle interaction on the classical integrability of a system of two masses moving inside a $d$-dimensional billiard. We find, in general, that the motion is strongly chaotic (typically exhibiting 'soft' chaos, with a mixed dynamics, where regions of phase space are still periodic or quasi-periodic), and with a strong dependence on the characteristic interaction length and strength. As perhaps one of the simplest examples (see Ref. [16 for a description of the general case), consider two particles of equal masses moving

in a $1 \mathrm{D}$ box defined in the interval $q:\left(-\frac{1}{2}, \frac{1}{2}\right)$ (we measure all lengths in terms of the box 
size). The particles are assumed to interact via a screened potential $V\left(q_{1}, q_{2}\right)=\exp \left(-\lambda \mid q_{1}-\right.$ $\left.q_{2} \mid\right) /\left|q_{1}-q_{2}\right|$, where $\lambda$ is the inverse screening length. Notice that this potential goes to a hard-core $\delta$-function when $\lambda \rightarrow \infty$, and the particles behave then as non-interacting but impenetrable points. In that case, the dynamics can be integrable in special cases of the mass ratios [17]. In this sense, $\lambda$ plays the role of a perturbation parameter which changes the degree of integrability of the system, as it determines the effective 'radius' of the particles for a given total energy. Direct calculations show that this is indeed the case.

The problem could in principle be solved by direct integration of the equations of motion, but we find convenient to transform it to a set of center-of-mass and relative coordinates $R=\left(q_{1}+q_{2}\right) / M$, and $r=q_{2}-q_{1}$, respectively, where the total mass $M=2$, and the reduced mass $\mu=1 / 2$. These equations define a two-dimensional space of coordinates $\rho=(r, R)$. In this space, we have a new set of equations for the boundary of the billiard, say $F_{j}(\rho)$. The Hamilton equations are transformed to $\dot{r}=p / \mu, \dot{R}=P / M$, and

$$
\begin{aligned}
& \dot{p}=-\nabla_{r} V(r)+\sum_{j} A_{j}(p, P) \delta\left[F_{j}(r, R)\right] \\
& \dot{P}=\sum_{j} B_{j}(p, P) \delta\left[F_{j}(r, R)\right] .
\end{aligned}
$$

The functions $A_{j}$ and $B_{j}$ describe the change in the momenta $p$ and $P$, due to the bounce on the $j$-th wall. Notice that these equations describe the motion of a single 'hyperparticle' in the two-dimensional $\rho$-space. This hyperbilliard description can be generalized to any number of dimensions [16].

Notice that bounces of the hyperparticle in the hyperbilliard correspond to bounces of the masses in the real/original dot. The walls of the billiard cause the breaking of translational symmetry of the system, and as a consequence, the center-of-mass (CM) momentum is no longer a constant of motion. In the case of non-interacting and equal-mass particles, the changes in the CM momentum are determined only by the geometry of the billiard. In our case, however, the interaction couples the CM and relative momenta after each bounce, which in turn depend on the momenta of each of the original masses.

Dynamical map. - We should also mention that these equations in $\rho$-space provide an 
interesting description which is also extremely useful: In between bounces, the hyper-particle moves freely along the $R$-axis, whereas the interaction acts only along $r$. These two motions are independent, and only become correlated at each bounce, when the different momentum components are changed, while keeping the total energy constant. Understanding this fact allows one to describe the motion in terms of a dynamical map connecting the different bounces. If the coordinate of the hyperparticle is $\rho_{n}=\left(r_{n}, R_{n}\right)$ at the time of the $n$-th bounce, then the time spent until the next bounce on the $j$-th wall is obviously the same along both components, and one can then write $\tau_{r}\left(\rho_{n}, \rho_{n+1}\right)=\tau_{R}\left(\rho_{n}, \rho_{n+1}\right)$. Since the CM motion is that of a free particle, except for the collisions with the walls, $\tau_{R}$ can be calculated simply. Similarly, for a pure Coulomb potential $(\lambda=0), \tau_{r}$ can be calculated analytically, so that the previous equation can be written in a more explicit form, $\tau\left\{T_{\epsilon}\left(\rho_{n}\right), T_{\epsilon}\left(\rho_{n+1}\right)\right\}=\mid$ $\left(R_{n+1}-R_{n}\right) /(P / M) \mid$, where $\tau$ is now the time elapsed going from $\rho_{n}$ to $\rho_{n+1}$, expressed in terms of the time $T_{\epsilon}(\rho)$ spent by the particle from the turning point to $\rho, T_{\epsilon}(\rho)=$ $\frac{r p}{2 \epsilon}+\left(\frac{\mu}{2 \epsilon^{3}}\right)^{1 / 2} \cosh ^{-1}(r \epsilon)^{1 / 2}$. Here $\epsilon=E-P^{2} / 2 M$, represents the energy left for the relative motion, as $E$ is the total energy of the two-mass system. For a weakly screened potential, $\lambda \leq 1$, we can expand $V(r)$ to first order and obtain a similar expression, where $E$ is scaled to $E-\lambda$. What follows, after these definitions is to characterize all the different possible trajectories in the triangular region in $\rho$-space. A simple algorithm can then be obtained to determine the Poincaré surfaces of section. This nontrivial (and clearly nonlinear) algebraic map provides then a full description of the dynamics. Its use (in lieu of the direct integration of the equations of motion) simplifies calculations a great deal, and allows one to better characterize the system, as we describe below.

Poincaré sections. - In order to characterize the motion of the two interacting particles (or the hyperparticle with its many degrees of freedom), we explore Poincaré sections of the resulting phase space. Having a four-dimensional space in this case $(r, R, p, P)$, we select to show those where one of the particles is at one end of the box (notice that the energy is a constant of motion here). Figure 1 shows a typical Poincaré section for $\lambda=0.1$ and $E=1.5$, obtained using the map described above (and which is virtually identical to the 
one obtained directly from integration of the equations of motion [16]). Notice that chaotic trajectories nearly fill the available phase space (for this given total energy). Increasing values of $\lambda$ give chaotic orbits that fill more of the available phase space. Moreover, there are also a number of islands of stability, as expected from the KAM theorem [1], near the fixed point corresponding to periodic symmetric motion in the non-interacting system (and indicated in the figure with a cross on the right axis). In fact, for all values of $E$ and $\lambda$, the

initial condition where $q_{1}(0)=-q_{2}(0)=\frac{1}{2}$, and $p_{1}(0)=-p_{2}(0)=\sqrt{E-V(1)}$ gives rise to a periodic orbit. Other islands also appear purely due to the interaction, as can be seen near $q_{2}=0.2, p_{2}=0$, and are associated with a sort of correlated motion of the two masses.

General values of $E$ and $\lambda$ give rise to this type of mixed dynamics, with chaotic and regular trajectories sharing the available phase space. This increasing degree of 'soft chaos' in the system (as $\lambda$ increases, for example), should be reflected in the level statistics of the corresponding quantum mechanical system, as we describe explicitly elsewhere in these proceedings. One naturally expects that this effect of interactions - turning a regular system into a non-integrable one — would be rather pervasive, regardless of the type of particle confinement and details of the interactions. In the following section we illustrate this effect in a somewhat different quantum mechanical model of a quantum dot.

\section{A QUANTUM DOT}

Here, we will use a model of a quantum dot which has been very successful in the description of recent experiments in these structures [6, 15,18]. The quantum dot is modeled as a parabolic potential well with circular symmetry, and the frequency (or curvature) is chosen to fit characteristic single-particle excitation energies in these devices. In this case, the few-particle problem, which includes fully the effect of interactions, can be solved quite accurately (numerically 'exactly') even in the presence of magnetic fields. The approach is based on a canonical (Jacobi) transformation to a set of auxiliary harmonic oscillator generating operators which allow one to write the interaction matrix elements in a closed 
analytical form, easily calculable [19]. This, together with the ability to separate the center of mass $(\mathrm{CM})$ degree of freedom from the 'relative' ones (a feature almost exclusive to the parabolic confinement potential), allows one to completely characterize and solve for the spectrum of up to three electrons in this parabolic well. Correspondingly, the spectrum can be characterized by $E=\varepsilon\left(N_{C M}, M_{C M}\right)+E_{\text {rel }}$, where the first term gives the CM manifold, and $E_{\text {rel }}$ is obtained from the relative-motion part of the Hamiltonian. In the case of three particles, this latter part has diagonal elements given by $\varepsilon\left(N_{-}, M_{-}\right)+\varepsilon\left(N_{+}, M_{+}\right)$, which are mixed by the Coulomb interaction (taken here as given by $V\left(r_{i j}\right)=e^{2} /\left(\epsilon r_{i j}\right)$, with $\epsilon$ a background dielectric constant which would be assumed variable in the results section below). Notice that in all these expressions,

$$
\varepsilon(N, M)=\hbar \Omega_{+}\left(N+\frac{1}{2}\right)+\hbar \Omega_{-}\left(M+\frac{1}{2}\right),
$$

arise from the auxiliary harmonic oscillators introduced in the canonical transformation [19], with $\Omega_{ \pm}=\left[\sqrt{4 \Omega_{0}^{2}+\omega_{c}^{2}} \pm \omega_{c}\right] / 2$. Here, $\Omega_{0}$ characterizes the single-particle harmonic confinement potential of the quantum dot (with a typical value of $\hbar \Omega_{0} \approx 1-2 \mathrm{meV}$ ), and $\omega_{c}=e B / m c$ is the cyclotron frequency of the electron. Due to the rotational symmetry of the system, the Coulomb interaction only couples states with the same relative angular momentum, given by $L_{\text {rel }}=\hbar\left(M_{+}+M_{-}-N_{+}-N_{-}\right)$. This is important, for apart from making the calculation simpler, it also allows us to carry out matrix diagonalizations with extremely high accuracy and for very many eigenstates (typically one to two thousand levels). This complete convergence is obviously important if one is interested in the analysis of the level spacings, as we now proceed to do.

Level statistics results. - The statistical analysis of the levels obtained as described above for three particles can be carried out according to the typical prescriptions in the literature. The level spectrum is used to extract a slowly-varying density of states with a smooth energy dependence, characteristic of the system at hand. This unfolding procedure then leaves one to study the structure of the level fluctuations of the spectrum, on which a number of statistical tests and statements can be made [2, [1]. The unfolding here is performed 
by defining the 'staircase' function $N(E)$ which gives the cumulative number of states below $E$, and then fitting this to a smooth polynomial (typically of fourth degree), $\tilde{N}(E)$. The 'linearized' or unfolded level sequence is then obtained from $x_{i}=\tilde{N}\left(E_{i}\right)$, where $E_{i}$ is the original sequence. This process, although not unique, gives similar results to other unfolding procedures (see [11] for a good discussion).

One can perform a number of statistical analyses. Here we focus on the nearest-neighbor spacings (NNS), obtained from $s_{i}=x_{i}-x_{i-1}$, and calculate the probability density function $P(s)$ for a given sequence. It is useful to define the integrated probability function [20], $I(s)=\int_{0}^{s} d s^{\prime} P\left(s^{\prime}\right)$. Notice that $I(s)$ is nothing but the total number of NNS below a given $s$, and can be uniquely calculated, without any dependence on the specific binning used to calculate the typical histogram representations of $P(s)$. In what follows, we use $I(s)$ to make quantitative statements, but revert to showing the more conventional $P(s)$.

Figure 2 shows a sequence of $N(E)$ staircase curves for different values of the background dielectric constant $\epsilon$, defined above. The sequences analyzed have all the same value of relative angular momentum, $L_{\mathrm{rel}}=3 \hbar$, and total spin $S=3 \hbar / 2$, and for a magnetic field value of $B=3 \mathrm{~T}$. Increasing values of $\epsilon$ would produce a progressively weaker value of the Coulomb interaction, and vice versa. We have varied $\epsilon$ such that the interaction varies by up to a factor of 50. In the figure, the curve labeled 1 corresponds to the value of $\epsilon=\epsilon_{1}=12.5$, found in GaAs, where most quantum dots are defined. Increasing the effective Coulomb interaction (with $\epsilon=\epsilon_{1} / 10$ ), produces the smoothest staircase function (labeled 10), completely devoid of the harmonic oscillator 'steps' in weaker interactions (as seen here for relative interaction strength $1 / 5$ and $1 / 3)$. One expects that for interaction 10 , the level mixing produced would be substantial, resulting in a level structure well described by the RMT distributions, as seen in other systems. We find that this is not the case here.

In fact, if one analyzes the appropriate histograms for the NNS distribution function $P(s)$, the interaction strength shows its effect quite clearly, as shown in Fig. 3. There, we show the corresponding $P(s)$ for three different interaction strength (or $\epsilon$ ) values. The anticipated Poisson distribution function one obtains for a 'generic' integrable system [2, 1] is similar to 
the case for interaction $1 / 3$. However, notice that this $P$ here goes to zero even faster than the expected Poisson form, reflecting the non-generic character of the delta-function-like distribution of the pure harmonic oscillator system. Moreover, as the interaction becomes stronger, notice that the distribution functions have a maximum not at zero $s$, but rather at a finite value. This behavior is more in agreement with the RMT predictions, where $P$ would be expected to be given by a GUE function (given the finite magnetic field), where $P(s \approx 0) \propto s^{2}$. One should notice, however, that in all cases we have studied, $P(s)$ never fully reaches the anticipated GUE for a fully chaotic system. The reason for this lack of full crossover into the GUE distribution is perhaps associated with the mixed dynamics of the corresponding classical system. In such cases, it has been argued that the level distribution can be seen as a superposition of Poisson and GUE functions, with weights corresponding to the coverage in phase space for the integrable and non-integrable regions, respectively [20]. At this point, however, we have not analyzed the classical system that directly models this quantum dot, and expect to report on this relation elsewhere. However, it may also be the case that this incomplete crossover to a GUE arises from the peculiar non-generic non-Poissonian distribution function of the integrable (non-interacting) system composed by overlapping harmonic oscillators, and/or remnant hidden symmetries. We have also fitted the $I$-functions to the well known Brody distribution, $I_{\text {Brody }}=1-\beta_{\alpha} \exp \left(-s^{\alpha+1}\right)$, providing a (phenomenological) measure of the crossover ( $\alpha=0$ for Poisson, $\alpha=1$ for GOE). In Fig. 2 and for unit interaction strength, we get $\alpha=0.04$, while $\alpha=0.25$ for interaction 10 .

Furthermore, the non-typical character of the level distribution in the harmonic oscillator is also reflected in the magnetic field dependence [21]. If one studies $P(s)$ for a given interaction strength but increasing magnetic fields, the system has a non-monotonic evolution. Examples of this behavior are shown in Ref. [21]. For low magnetic field $(B=0.3 \mathrm{~T}$, and 'unit' interaction, with $\left.\epsilon=\epsilon_{1}\right), P(s)$ is not the harmonic oscillator delta-like function, but is somewhat shifted towards a GUE (Brody-fit with $\alpha \approx 0.1$ ). As the field increases, however, the $P$-distribution reaches a 'maximum' $\operatorname{crossover}(\alpha \approx 0.2$, for $B=1 \mathrm{~T})$, before going back towards a more delta-like function, as seen for $B=10 \mathrm{~T}$, which drops faster than 
a Poissonian distribution. That this occurs is understandable, since for high magnetic fields one would expect to reach a regime where the interactions would be a weak perturbation (for a given value of $\epsilon$ ), and the spectrum would evolve towards a set of Landau levels. This non-monotonic behavior is seen in all the level sequences we have studied.

\section{CONCLUSIONS}

We have shown that the interactions introduce classical non-integrability in a quantumdot system, even if the geometry is integrable for the one-particle problem. Moreover, this behavior, given the Bohigas et al. conjecture [1], would be expected to be reflected in the accompanying level structure of the corresponding quantum mechanical version of the system. We have found, that as we introduce interactions, indeed the NNS fluctuation distribution function exhibits an apparent crossover towards one of the RMT functions, with a characteristic maximum away from zero spacing. This crossover, is found to be not complete, however, even for rather strong interaction, perhaps due to the mixed dynamics in the classical counterpart. The NNS distribution exhibits non-monotonic magnetic field dependence, associated with the fact that in that regime the particle interactions are but a weak perturbation of the Landau level spectrum. A number of theoretical questions still remain, however, as more direct comparison of the classical and quantum systems is needed, perhaps including a study of the associated wave functions and possible appearance of 'scars' [4]. Nevertheless, the importance of interactions in the details of level structure and associated experiments, as discussed in the introduction, cannot be ignored. We hope that this, as well as semiclassical treatments (see Richter's article in these proceedings), motivate further understanding of this fascinating problem.

Acknowledgments. This work was supported in part by US DOE grant no. DEFG02-91ER45334, CONACyT-México, and the Deutsche Forschungsgemeinschaft. SEU also acknowledges support from the A. von Humboldt Stiftung. 


\section{REFERENCES}

[1] M.C. Gutzwiller, Chaos in Classical and Quantum Mechanics (Springer, New York, 1990).

[2] M.L. Mehta, Random Matrices (Academic, Boston, 1991).

[3] L.P. Gorkov and G.M. Eliashberg, Sov. Phys. JETP 21, 940 (1965); K.B. Efetov, Adv. Phys. 32, 53 (1983); B.L. Altshuler and B.Z. Spivak, Sov. Phys. JETP 65, 343 (1987); Y.H. Zheng and R.A. Serota, Phys. Rev. B 50, 2492 (1994).

[4] E.J. Heller, Phys. Rev. Lett. 53, 1515 (1984).

[5] H. Bruus, C.H. Lewenkopf, and E.R. Mucciolo, Phys. Rev. B 53, 9968 (1996); R. Akis, D.K. Ferry, and J.P. Bird, Phys. Rev. Lett. 79, 123 (1997).

[6] D. Weiss, K. Richter, E. Vasiliadou, and G. Lütjering, Surf. Sci. 305, 408 (1994); see also article by K. Ensslin in these proceedings.

[7] G. Petschel and T. Geisel, Phys. Rev. Lett. 71, 239 (1993); T. Geisel, R. Ketzmerick, and G. Petschel, Phys. Rev. Lett. 66, 1651 (1991).

[8] O. Steffens, M. Suhrke, and P. Rotter, Phys. Rev. B 55, 4486 (1997).

[9] R.A. Jalabert, A.D. Stone, and Y. Alhassid, Phys. Rev. Lett. 68, 3468 (1992).

[10] C. M. Marcus et al., Phys. Rev. Lett. 69, 506 (1992); A. Chang et al., Phys. Rev. Lett. 73, 2111 (1994); J. P. Bird et al., Phys. Rev. B 50, 18678 (1994).

[11] R. Berkovits and Y. Avishai, J. Phys. Cond. Matt. 8, 389 (1996); H. Bruus and J.-C. Anglès d'Auriac, Phys. Rev. B 55, 9142 (1997).

[12] L. Viña, M. Potemski, and W.I. Wang, Proceed. of 23rd ICPS, M. Schaeffler and R. Zimmermann, eds. (World Scientific, Singapore, 1996), p. 2047.

[13] U. Sivan et al., Phys. Rev. Lett. 77, 1123 (1996); F. Simmel, T. Heinzel, and D.A. 
Wharam, Europhys. Lett. 38, 123 (1997).

[14] A.T. Johnson et al., Phys. Rev. Lett. 69, 1592 (1992); J. Weis. R. Haug, K.v. Klitzing, and K. Ploog, Phys. Rev. Lett. 71, 4019 (1993).

[15] D. Pfannkuche and S.E. Ulloa, Advances in Solid State Physics 35, 65 (Vieweg, Braunschweig/Wiesbaden, 1996); Phys. Rev. Lett. 74, 1194 (1995); Superlatt. Microstr. 15, 269 (1994).

[16] L. Meza-Montes and S.E. Ulloa, Phys. Rev. E 55, 6315 (1997).

[17] Ya. G. Sinai, Introduction to Ergodic Theory, Mathematical Notes Vol. 18 (Princeton University Press, 1976).

[18] K. Jauregui, W. Häusler, D. Weinmann, and B. Kramer, Phys. Rev. B 53, R1713 (1996).

[19] P. Hawrylak and D. Pfannkuche, Phys. Rev. Lett. 70, 485 (1993).

[20] M.V. Berry and M. Robnick, J. Phys. A: Math. Gen. 17, 2413 (1984); T. Prosen and M. Robnick, ibid 26, 2371 (1993).

[21] S.E. Ulloa and D. Pfannkuche, Superlatt. Microstr. 21, 21 (1997). 


\section{FIGURES}

FIG. 1. Poincaré section of two-particles in a box with interaction given by $\lambda=0.1$. Total energy $E=1.5$, and $q_{1}=-\frac{1}{2}$.

FIG. 2. Typical staircase function for different interaction strengths. For interaction strength 10, the harmonic oscillator structure is completely lost, while it is visible for weak interaction $(1 / 3$ and $1 / 5)$.

FIG. 3. NNS distribution function for varying interaction strength values (in units of the interaction in GaAs). Notice evolution towards a GUE distribution for stronger interaction.

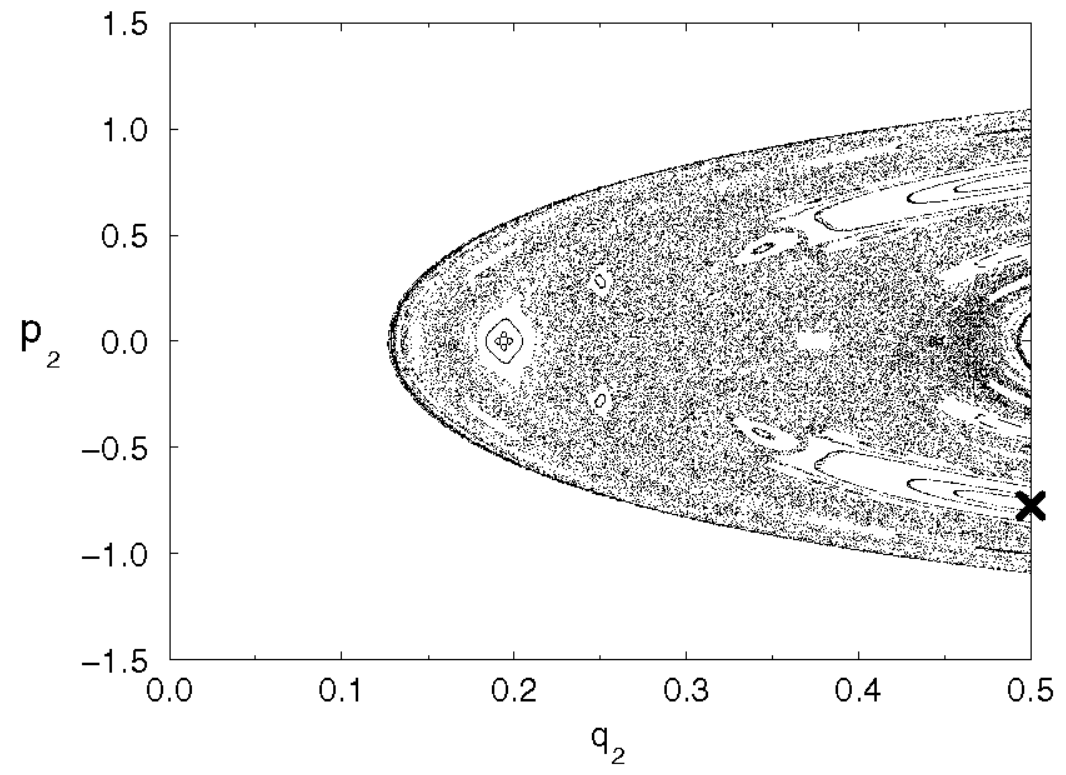

\section{FIG 1}


$T$
$\mathbb{2}$
$N$

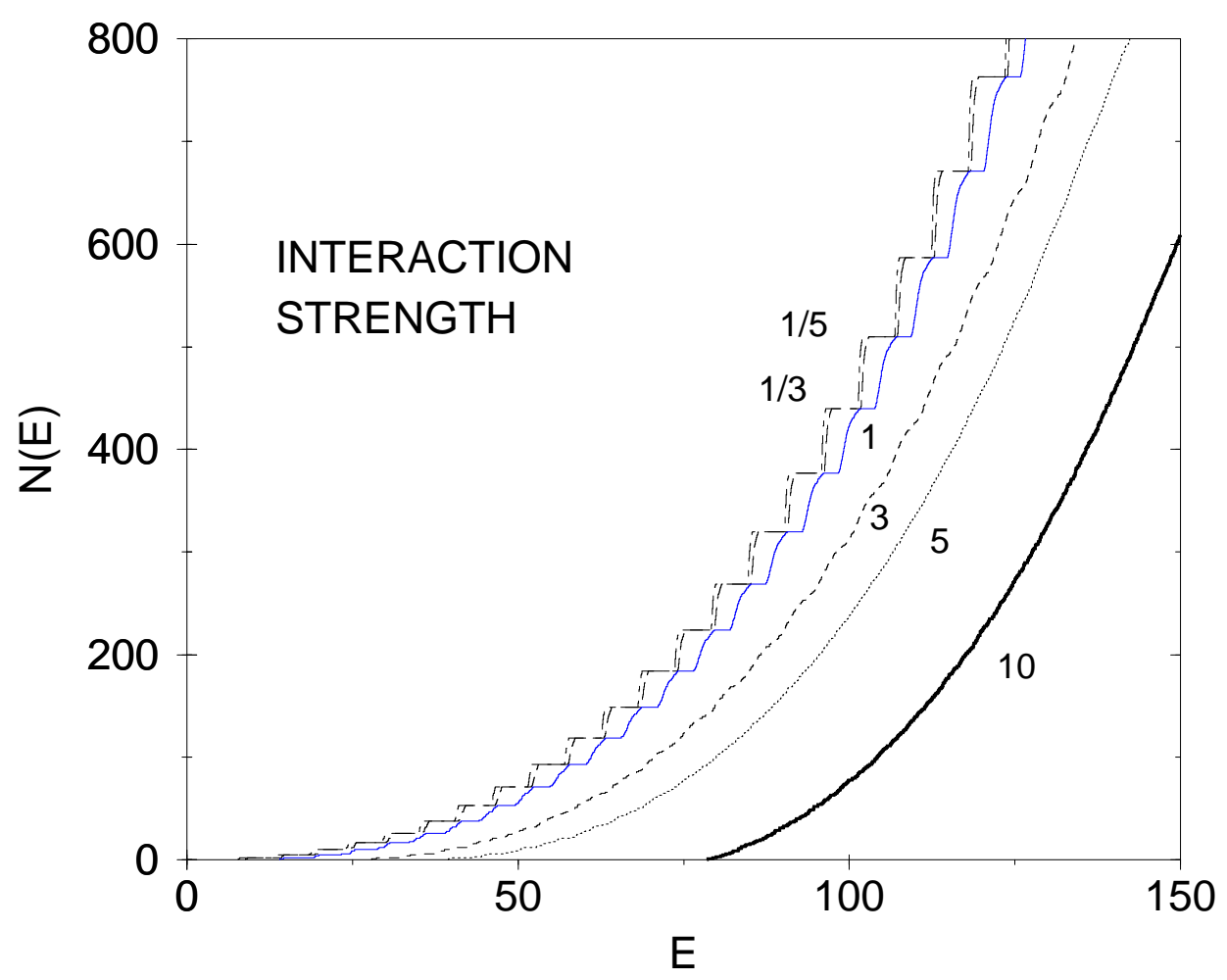




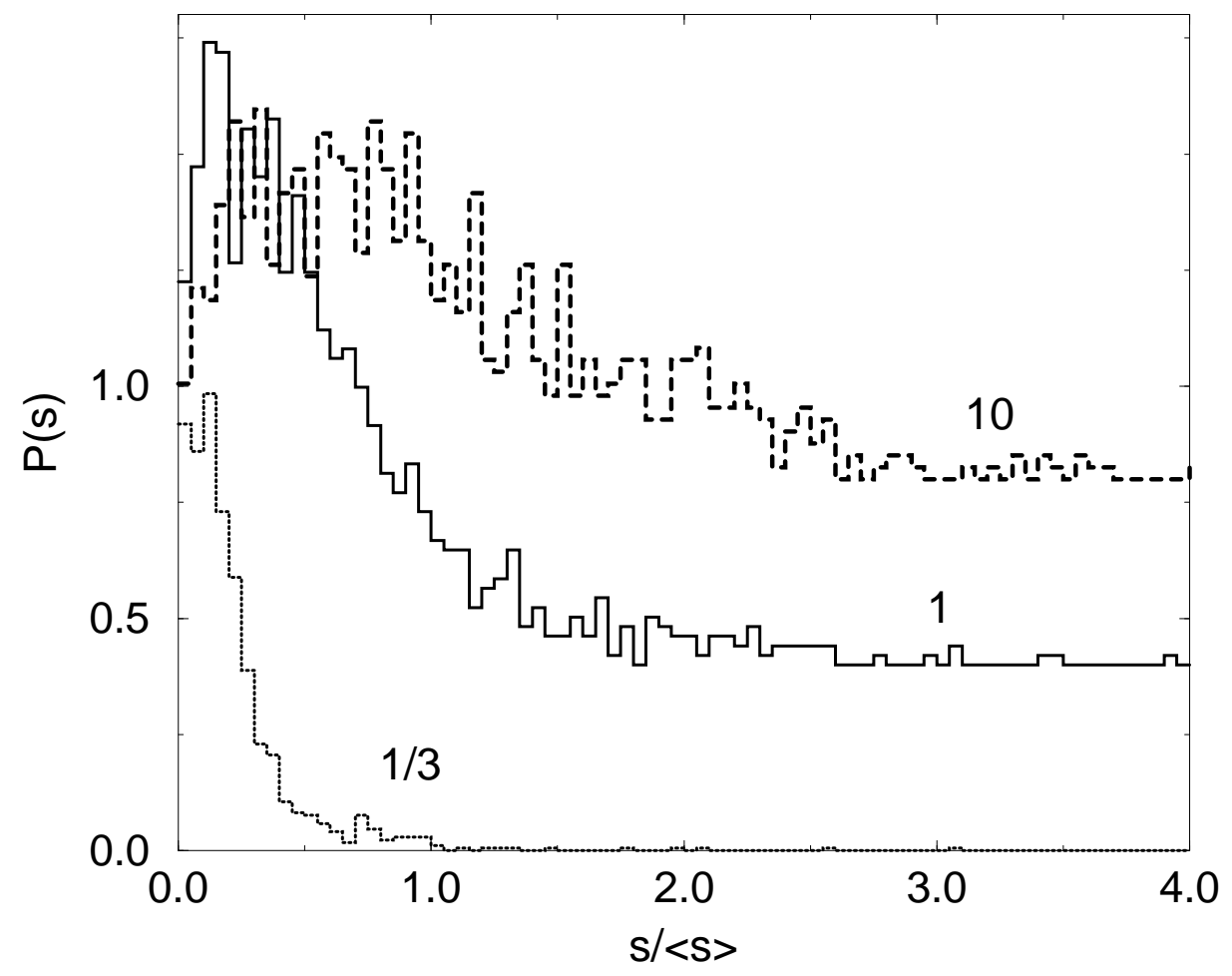

